\title{
A Large TPC Prototype for a Linear Collider Detector
}

\author{
Peter Schade $^{\mathrm{a}}$, Jochen Kaminski ${ }^{\mathrm{b}}$, for the LCTPC collaboration \\ ${ }^{a}$ DESY, Hamburg, Germany \\ ${ }^{b}$ University of Bonn, Bonn, Germany
}

\begin{abstract}
A Time Projection Chamber (TPC), operated in a detector at a future linear $e^{+} e^{-}$collider, will require a significantly advanced readout concept compared to the traditional pad-wire-based systems used previously. The LCTPC collaboration studies the prospects of TPC readout systems based on Micro Pattern Gas Detectors, namely GEMs and Micromegas, in combination with pads or a CMOS on the underlying anode plane. In small TPC prototype chambers, resolutions of better than $\sigma_{\perp}=100 \mu \mathrm{m}$ were demonstrated for drift distances of up to $66 \mathrm{~cm}$. In the future, the R\&D efforts will focus on the technical realization of large-surface readout systems with the aim to develop a technical concept for a readout module of a future TPC. A Large TPC Prototype with a diameter of $77 \mathrm{~cm}$ has been constructed for the planned studies. It is part of a test beam infrastructure at the electron test beam at DESY. First test beam operations with Micromegas and GEM based prototype modules in December 2008 and during 2009 gave promising results.
\end{abstract}

Key words: ILC, ILD, Time Projection Chamber, LCTPC, Large Prototype, GEM, Micromegas, CLIC

\section{Introduction}

Currently two concepts for a linear $e^{+} e^{-}$collider are under study, the International Linear Collider, ILC [1], and the Compact Linear Collider, CLIC [2]. The physics program of an $e^{+} e^{-}$ collider with a center-of-mass energy in the $\mathrm{TeV}$ regime will complement the program of the Large Hadron Collider and is well documented, e.g. in [3].

In parallel to the machine designs, detector concepts are being prepared. Recently the International Large Detector concept, ILD [4], has been validated by an independent advisory committee [5]. This detector concept is optimized for particle flow reconstruction [6].

Particle flow requires high granular calorimetry in combination with very efficient and hermetic tracking, and a reliable particle identification. For ILD, a Time Projection Chamber is proposed as the main tracking detector. This TPC will measure up to 200 space points per track, if operated with a pad readout, and thus provide a very high tracking efficiency and robustness with respect to machine backgrounds. In addition, the specific energy loss of charged particles in the TPC, $\mathrm{d} E / \mathrm{d} x$, can be measured and used as an input to the particle identification.

The performance goals of the ILD TPC are defined in the ILD Letter of Intent [4] (Tab. 1) and exceed the corresponding performances reached by previous TPCs in collider experiments by up to one order of magnitude. Therefore, a significant advancement of traditional TPC readout structures is mandatory.

The Linear Collider TPC collaboration (LCTPC) is pursuing R\&D activities to determine the best state-of-the-art technology, which can satisfy the ILD TPC resolution goals, allows the construction of a sufficiently lightweight end plate including the electronics, and offers a stable operation. Under consideration are Micro Pattern Gas Detectors (MPGD), namely Gas

\begin{tabular}{ll}
\hline Size & $\begin{array}{l}\text { outer field cage } \varnothing: 3.6 \mathrm{~m} \\
\text { total length: } 4.3 \mathrm{~m}\end{array}$ \\
& $\sigma_{\perp}<100 \mu \mathrm{m}$ modulo $\varphi$ \\
point resolution in $r \varphi$ & $\sigma_{z}<0.5 \mathrm{~mm}$ modulo $\theta$ \\
point resolution in $z$ & $\delta\left(1 / p_{\perp}\right) \approx 9 \cdot 10^{-5} \mathrm{GeV}^{-1}$ \\
momentum res. (TPC alone) & $\sim 5 \%$ \\
$\mathrm{~d} E / \mathrm{d} x$ resolution & $\lesssim 0.01 \mathrm{X}_{0}$ of the inner barrel \\
TPC material budget & $\lesssim 0.04 \mathrm{X}_{0}$ to the outer barrel \\
& $\lesssim 0.15 \mathrm{X}_{0}$ to the end caps in $z$ \\
efficiency (TPC alone) & $>97 \%$ for $\left.\mathrm{p}_{t}>1 \mathrm{GeV} / \mathrm{c}\right)$ \\
\hline
\end{tabular}

Table 1: Design goals for the ILD TPC [4]

Electron Multipliers (GEMs) [7] or Micro Mesh Gas amplifiers (Micromegas) [8], combined with a pad or a pixel readout.

\section{Feasibility Studies for an MPGD based TPC Readout}

Initial feasibility studies of a MPGD based TPC readout have been performed by different research groups inside LCTPC. For the demonstration of the principle, GEM and Micromegas structures measuring $10 \times 10 \mathrm{~cm}^{2}$ were operated in small TPC prototypes with diameters of up to $30 \mathrm{~cm}$. As an example of the variety of results obtained, two setups with GEM readout are presented in the following (for more results, including Micromegas, see references in [4]).

\subsection{GEMs with Pad Readout}

A GEM readout system in combination with pads of dimensions $1.27 \times 7 \mathrm{~mm}^{2}$ was operated in the MediTPC prototype at DESY. This prototype has an inner diameter of $25 \mathrm{~cm}$ (Fig. 1). Figure 2 shows the resolution measured in a $4 \mathrm{~T}$ magnetic field 


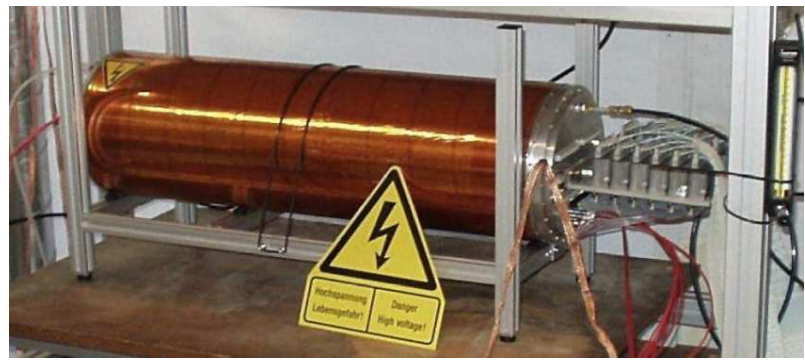

Figure 1: MediTPC prototype detector at DESY

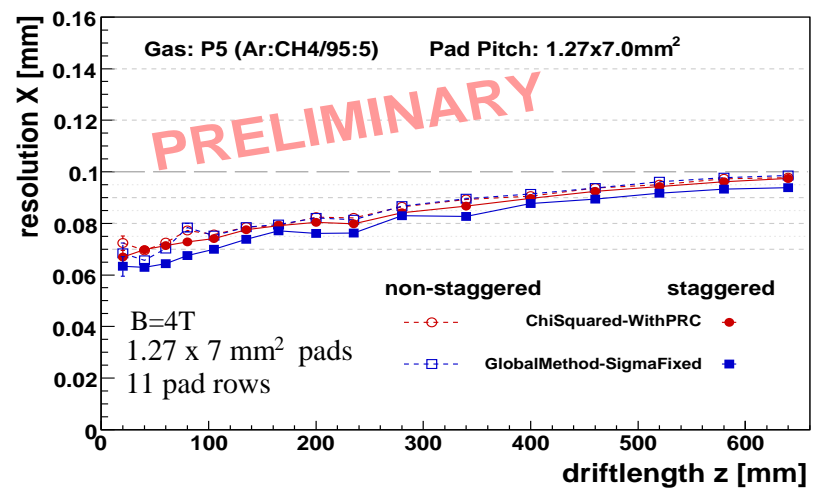

Figure 2: Point resolution measured with the MediTPC and pad readout

with a triple GEM amplification structure. Cosmic muons were used as a signal source for the data taking.

Two methods were used for the reconstruction of trajectories, a $\chi^{2}$ and a likelihood fit $[9,10]$. The evaluated transverse spatial resolutions stay below the limit of $100 \mu \mathrm{m}$ for the maximum drift length of the chamber of $66 \mathrm{~cm}$ and are independent of the reconstruction method.

\subsection{GEMs with Pixel Readout}

To fully exploit the potential of Micro-Pattern Gas Detectors, a new readout concept is studied within LCTPC. A highly pixelized readout ASIC is placed directly below the last gas amplification stage. The bump bonding pads of the readout chip are used to collect the electron signal. The Timepix-chip [11] is currently best suited for this application. It features $256 \times 256$ pixels with a pitch of $55 \times 55 \mu \mathrm{m}^{2}$. Each pixel can measure either the arrival time or the charge.

A prototype detector with a drift length of $26 \mathrm{~cm}$ was constructed at Bonn (Fig. 3). It is equipped with a stack of three GEMs and a Timepix chip. Cosmic rays were used in this setup to study the tracking characteristics and Figure 4 shows the measured transverse spatial resolution of the reconstructed hits. It deviates only very little from the single electron diffusion limit. Several variables such as size and charge in dependence on the drift distance indicate that for drift distances above $10 \mathrm{~cm}$ the charge depositions are created by single primary electrons. For shorter drift distances the diffusion does not separate the electrons sufficiently and the charge depositions can originate from more than one primary charge (for details see [12]). Due to the high number of primary electrons a very good overall performance of the TPC can be expected.

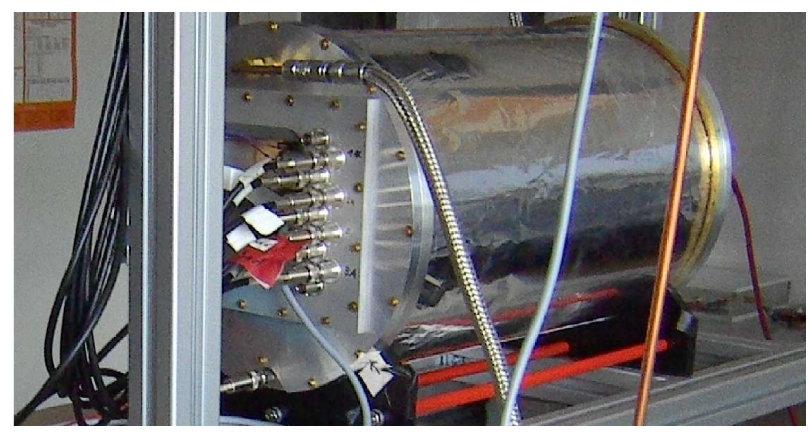

Figure 3: Prototype detector at Bonn

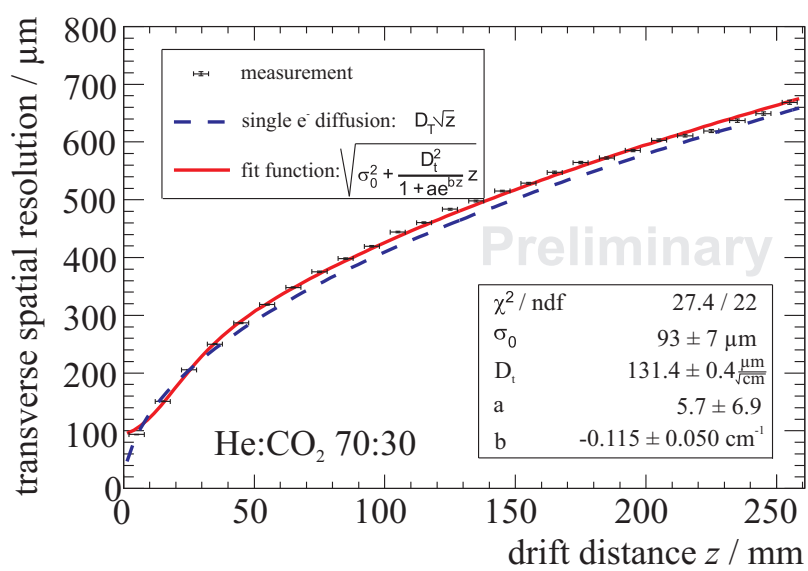

Figure 4: Point resolution measured with a Timepix chip

\section{The TPC R\&D Test-Beam Setup at DESY}

In the next step of R\&D work for a TPC of a future linear collider detector, it is foreseen to realize large surface MPGD readout structures with appropriate electronics. A TPC test setup for these studies has been realized with the help of the EUDET project [13]. It is installed at the DESY $6 \mathrm{GeV}$ electron test beam. The main components are a superconducting magnet, called PCMAG, and a Large TPC Prototype (LP). In addition, a muon hodoscope has been installed around the TPC to perform runs with cosmic particles. A set of highly accurate Si-strip modules completes the setup.

The magnetic field of PCMAG was measured precisely in 2007 [14]. It has a strength of up to $1.25 \mathrm{~T}$. The material budget of the magnet is about $20 \% \mathrm{X}_{0}$ per wall, and its bore diameter is $85 \mathrm{~cm}$. A support structure is installed inside the magnet which holds the LP and the silicon modules. In operation, test beam electrons pass the magnet and create tracks in the sensitive volume of the LP. The silicon detectors provide two independent space points of the electron trajectories with a spatial resolution of $10 \mu \mathrm{m}$, directly outside the TPC (see [15]). These detectors were installed inside PCMAG in October 2009.

\subsection{The Large TPC Prototype}

The field cage for the Large TPC Prototype [16] (Fig. 5) has an outer diameter of $77 \mathrm{~cm}$, which is limited by the available space inside PCMAG, and a usable inner diameter of $72 \mathrm{~cm}$. Its structure is produced from lightweight composite materials, 


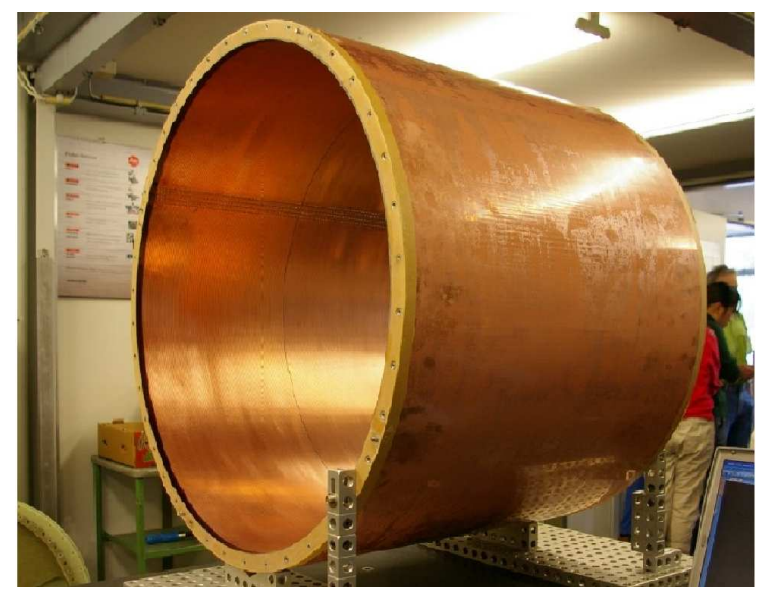

Figure 5: Field cage of the Large TPC Prototype

namely an aramid honeycomb embedded between two $300-\mu$ mthin layers of glass fiber reinforced plastics (GRP) (Fig. 6). This wall has an estimated material budget of $1.24 \% \mathrm{X}_{0}$ (Tab. 2). The goal of $1 \% \mathrm{X}_{0}$ per wall seems to be within reach, for example when the copper layers in the wall are replaced by aluminum ones and the GRP layers are thinned down. At the same time, the LP has a diameter similar to the one foreseen for the inner field cage of ILD $(\varnothing \approx 66 \mathrm{~cm})$.

In a current design proposal, it is foreseen to cover the ILD TPC end plates with modules shaped like ring segments. The first anode plate for the LP, constructed within LCTPC, has seven module windows to accommodate corresponding prototype readout modules (Fig. 7). The size of the windows is about $22 \mathrm{~cm} \times 17 \mathrm{~cm}$.

\section{Test Beam Operations with GEMs and Micromegas Modules with Pad Readout}

During test beam campaigns, the LP end plate is equipped successively with several Micromegas or GEM modules. Each time, up to several thousand of standard pads or CMOS pixels are read out. The first operation of the setup were performed in December 2008 using a single Micromegas module with 1728 pads, each measuring $3.2 \times 7 \mathrm{~mm}^{2}$. The rather broad pads were covered on the top with a homogeneous resistive layer, either a carbon loaded polyimide $(4-8 \mathrm{M} \Omega / \mathrm{sq})$ or a resistive ink $(1-4 \mathrm{M} \Omega / \mathrm{sq})$. The resistive layer spreads the charge over the pad plane, so that about 2-4 pads per row measure a signal of a trajectory. For the readout of the Micromegas module, the AFTER electronics [17] from the T2K experiment was used.

In a following test beam campaign, three GEM prototype modules were operated (Fig. 7). The readout system for the GEM modules was based on the electronics setup used by the ALICE experiment at the LHC, ALTRO. The ALTRO system has been adapted for the needs of the LP, namely the front-end cards were equipped with a new programmable charge sensitive preamplifier (PCA16) [18], developed at CERN. In 2009, only 3200 channels of ALTRO electronics were available, which was not sufficient to equip all of the 5152 channels per GEM mod-

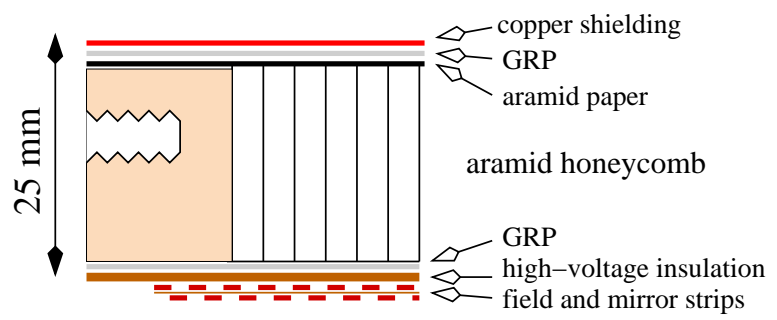

Figure 6: Wall structure of the Large Prototype field cage

\begin{tabular}{|c|c|c|}
\hline material & eff. thickness [cm] & $X / X_{0}[\%]$ \\
\hline copper & 0.007 & 0.48 \\
\hline polyimide & 0.017 & 0.06 \\
\hline glass fiber & 0.04 & 0.38 \\
\hline aramid paper & 0.007 & 0.02 \\
\hline honeycomb & 2.35 & 0.17 \\
\hline ероху & $\approx 0.06$ & $\approx 0.17$ \\
\hline
\end{tabular}

Table 2: Materials of the field cage wall (Fig. 6) and material budget estimation

ule. The GEM modules consisted of uniform pads of dimensions $1.2 \times 5.4 \mathrm{~mm}^{2}$. The channels were distributed over the three modules such, that trajectories with a maximum length of about $60 \mathrm{~cm}$ could be recorded.

In both cases, an $\mathrm{ArCF}_{4} \mathrm{iC}_{4} \mathrm{H}_{10}(95 / 3 / 2)$ gas mixture was used in the LP while the magnetic field strength was $1 \mathrm{~T}$.

The transverse spatial resolution $\sigma_{\perp}$ in a TPC is given by three contributions $[19,20]$ :

- a contribution $\sigma_{p a d}$ which rises for short drift distances of $z \lesssim 5 \mathrm{~cm}$ and is constant for larger $z$. It is caused by the finite pad width.

- a drift length dependent contribution which is dominated by the gas diffusion constant $D$ divided by an effective number electrons per pad row $N_{\text {eff }}$.

- a constant term $\sigma_{\text {ele }}$ caused by electronic noise.

Thus $\sigma_{\perp}^{2}=\sigma_{\text {pad }}^{2}+D^{2} / N_{\text {eff }} \cdot z+\sigma_{\text {ele }}^{2}$.

The measured resolutions in the GEM and Micromegas test beam campaigns (Fig. 8) are fitted with $\sigma_{\perp}=\left(\sigma_{0}^{2}+D^{2} / N_{e f f}\right.$. $z)^{0.5}$ for drift distances of $z>5 \mathrm{~cm}$. Here $\sigma_{0}$ combines the constant contributions $\sigma_{\text {ele }}$ and $\sigma_{\text {pad }}$. The values obtained from preliminary fits (Tab. 3) show a similar performance for both amplification technologies with pad readout. In the current setup, $\sigma_{\perp}$ rises above the limit of $100 \mu \mathrm{m}$ due to the diffusion, which is insufficiently suppressed in the $1 \mathrm{~T}$ field. However, the operations of small prototypes demonstrated that the impact of the diffusion can be kept moderate, if the TPC is operated in high magnetic fields of $3-4 \mathrm{~T}$. Thus the $100-\mu \mathrm{m}$ limit up to a drift distances of $2.15 \mathrm{~m}$ for the ILD seems to be within reach.

In addition, for drift distances longer than $30 \mathrm{~cm}$, magnetic field inhomogeneities of PCMAG, which were not yet taken into account in the track reconstruction, lead to a degradation of the resolution. The impact of the field inhomogeneities will be corrected in a future refined data analysis. 


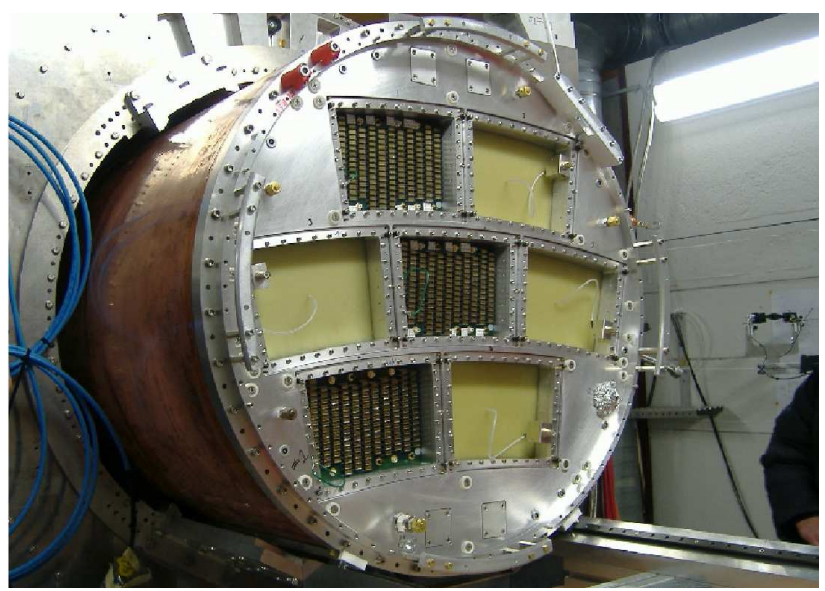

Figure 7: Large TPC Prototype in PCMAG with three GEM modules mounted on the anode end plate

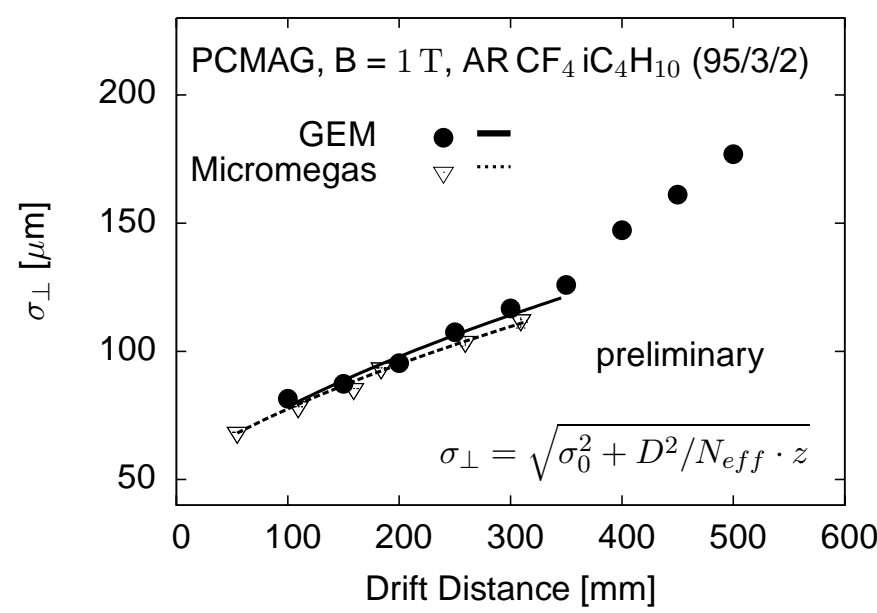

Figure 8: Resolution measured in the test beam campaigns with GEM and Micromegas modules [21]

\begin{tabular}{l|ccc} 
module & $\sigma_{0}[\mu \mathrm{m}]$ & $D[\mu \mathrm{m} / \sqrt{c m}]$ & $N_{\text {eff }}$ for $1 \mathrm{~cm}$ \\
\hline Micromegas & $54.8 \pm 1.6$ & & $45.4 \pm 2$ \\
GEM & $51.9 \pm 1.6$ & $92.7 \pm 0.4$ & $45.5 \pm 1$
\end{tabular}

Table 3: Parameters obtained for the preliminary fits in Fig. 8 [21] (for the expectation of $N_{\text {eff }}$ see [22])

\section{Test Beam Operations with Pixel Readout Systems}

The Timepix concept described in section 2.2 is also studied with the Large Prototype. A first module with a triple-GEM stack and eight Timepix chips was produced. During a test beam campaign in the summer of 2009, a very good behavior could be demonstrated in various gas mixtures and in a magnetic field of up to 1 T. Fig. 9 shows a track passing the ASICs. A second module with pixelized readout is in preparation. This time the gas amplification stage is not made of GEMs but of a Micromegas mesh, which has been produced on top of the Timepix chip with the help of post-processing methods [23]. Because of the reduced structure size compared to GEMs, a further improvement of the performance is possible.
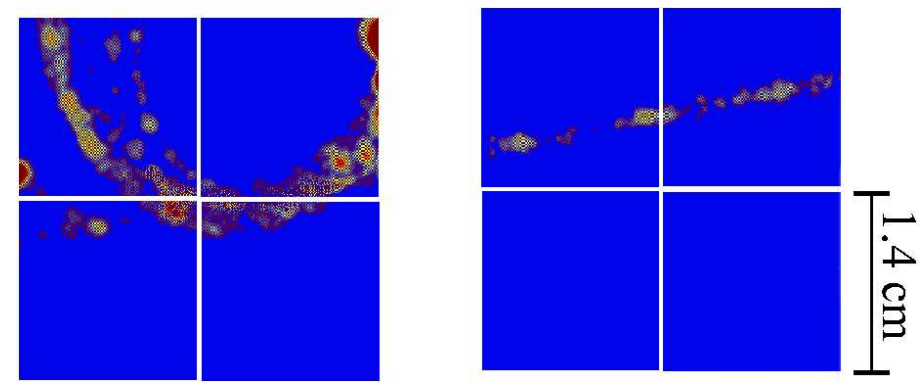

Figure 9: Track seen by the eight Timepix chips of the LP-module

\section{Summary and Outlook}

The Large Prototype (LP) is the first TPC test chamber constructed within LCTPC with a size similar to the inner field cage of the ILD TPC. Its construction demonstrated that a material budget of $1 \% \mathrm{X}_{0}$ per wall for the ILD seems possible.

In first test beam campaigns, GEM and Micromegas modules with pad readout were operated in the LP and transverse spatial resolutions of about $\sigma_{\perp}=60-70 \mu \mathrm{m}$ were observed for drift distances of the order $10 \mathrm{~cm}$. The current technologies offer the prospect to reach $\sigma_{\perp} \lesssim 100 \mu \mathrm{m}$ in the ILD TPC, which will be operated in a $3.5 \mathrm{~T}$ magnetic field.

Further test beam campaigns are planned for 2010, both with GEM and Micromegas modules. The goal is to operate the anode plate fully equipped with 7 pad readout modules at the end of the year. Also, further operations of LP modules with pixel readout are foreseen in 2010.

\section{References}

[1] J. Brau et al., ILC Collaboration, arXiv:0712.1950.

[2] R. Tomas, Phys. Rev. ST Accel. Beams 13 (2010) 014801.

[3] H. Murayama and M. E. Peskin, Ann. Rev. Nucl. Part. Sci. 46 (1996) 533.

[4] ILD Collaboration, ILD Letter of Intent, DESY 2009-87, KEK 2009-6.

[5] IDAG Report on the Validation of Letters of Intent for ILC detectors, Aug. 2009, http://www.linearcollider.org/physics-detectors/Detectors/IDAG

[6] M. A. Thomson, Nucl. Instr. and Meth. A 611 (2009) 25.

[7] F. Sauli, Nucl. Instr. and Meth. A 386 (1997) 531.

[8] Y. Giomataris, P. Rebourgeard, J. P. Robert and G. Charpak, Nucl. Instr. and Meth. A 376 (1996) 29.

[9] M. E. Janssen, DESY-THESIS-2008-011.

[10] D. Karlen, P. Poffenberger and G. Rosenbaum, Nucl. Instr. and Meth. A 555 (2005) 80.

[11] X. Llopart et al., Nucl. Instr. and Meth. A 581 (2007) 485.

[12] C. Brezina et al., JINST 4 (2009) P11015.

[13] http://www.eudet.org/, 2010.

[14] C. Grefe, DESY-THESIS-2008-052.

[15] S. Hänsel, Tests of a Prototype of the Silicon Tracking System of the ILD Concept, Nucl. Instr. and Meth. A - these proceedings (2010).

[16] P. Schade, DESY-THESIS-2009-040.

[17] P. Baron et al., IEEE Trans. Nucl. Sci. 55 (2008) 1744.

[18] L. Jonsson, U. Mjornmark, EUDET-Memo-2008-53.

[19] D. C. Arogancia et al., Nucl. Instr. and Meth. A 602 (2009) 403.

[20] Also talks at the ILC TPC School, Tsinghua University, Beijing, Jan. 711, 2008, http://www.hep.tsinghua.edu.cn/talks/TPCSchool2008/.

[21] A. Sugiyama, D. Attie, Talks at the LCTPC collaboration Meeting, DESY, Hamburg, Sept. 21, 2009, http://ilcagenda.linearcollider.org/conferenceDisplay.py?confId=3742

[22] M. Kobayashi, Nucl. Instr. and Meth. A 562 (2006) 136.

[23] Y. Bilevych et al., Nucl. Instr. and Meth. A 610 (2009) 644. 